\title{
FREQUENCY OF SARS-COV-2 INFECTION AND CHARACTERIZATION OF THE CLINICAL COURSE IN PATIENTS WITH SYSTEMIC LUPUS ERYTHEMATOSUS IN THE CITY OF MANAUS, AMAZONAS
}

Gabriella Bacellar Marques ${ }^{1}$, Lucila Cristina Tomé Garcia1,*, Cecília Tizatto Barroso ${ }^{1}$, Vitória Miki Pang Takatani ${ }^{1}$, Samuel Elias Basualto Dias ${ }^{1}$, Gustavo Lopes de Castro ${ }^{1}$, Clara Pinheiro Martins ${ }^{1}$, Sandra Lúcia Euzébio Ribeiro ${ }^{1}$

1.Universidade Federal do Amazonas, Manaus (AM), Brazil.

*Corresponding author: lucilanaoluciola@gmail.com

\section{BACKGROUND}

During the SARS-CoV-2 pandemic, it was interrogated whether systemic lupus erythematosus (SLE) patients with COVID-19 would be at risk of worse outcome due to chronic inflammatory autoimmune condition and use of immunosuppressants. The Amazon region receives intense ultraviolet radiation, factor that can influence high expression of SLE. With 415,846 cases of COVID-19 till September 2021, the state of Amazonas is an ideal environment to characterize the disease evolution in patients with SLE. This study seeks to describe the frequency and clinical course of COVID-19 in SLE patients followed at the Ambulatório Araújo Lima, in Manaus (AM).

\section{METHODS}

Prospective observational study, based on data analysis from SLE patients of Project Mário Pinotti II, organized by the Brazilian Society of Rheumatology, in Manaus, capital of Amazonas state. We analyzed: gender, age, flu-like symptoms (asthenia, headache, coryza, diarrhea, dysgeusia, dysosmia, dyspnea, cough, sore throat, fever, nausea, dizziness and vomiting) and clinical outcome. We used the clinical criteria established by the Brazilian Ministry of Health to define COVID-19 confirmed case: flu-like-symptoms case associated with olfactory or gustatory dysfunction without any other previous cause. The study was approved by the Ethics Committee.

\section{RESULTS}

A total of 271 SLE patients were followed through 6 telephone evaluations, with 14 days of interval, from 28 April to 30 September 2020. The mean age was $38.36 \pm 11.39$ years, 252 (92.99\%) were females and 19 (7.01\%) males. In the 1 st evaluation, $45(17.79 \%)$ had COVID-19, the main symptoms were dysgeusia, dysosmia and headache; in the 2nd evaluation, 8 (3.19\%) had COVID-19, the main symptoms dysgeusia, dysosmia and cough; in the 3rd evaluation, 2 (0.78\%) had COVID-19, the main symptom dysgeusia; in 4th evaluation, 5 (1.91\%) had COVID-19, the main symptoms dysgeusia, asthenia and fever; in the 5th evaluation, $3(1.12 \%)$ had COVID-19, the main symptoms dysgeusia, dysosmia, asthenia, headache and cough; in the 6th evaluation, 1 (0.37\%) had COVID-19, the main symptoms dysgeusia and dysosmia. In the 1st evaluation, 3 (8.89\%) of COVID-19 patients were hospitalized and, in the 2nd, $1(2.5 \%)$ was hospitalized. There was no need for intensive care or mechanical ventilation. There were no deaths.

\section{CONCLUSION}

Dysgeusia, dysosmia, headache, asthenia and cough were the most common symptoms among patients during the evaluations. Most cases were self-resolving, without need for hospitalization and no death. In this cohort, a high suspicion of COVID-19 infection was not uncommon, but with no severity.

\section{KEYWORDS}

Systemic lupus erythematosus, SARS-CoV-2, Amazon. 